\title{
First steps for integrating sex and gender considerations into basic experimental biomedical research
}

\author{
Stacey A. Ritz, ${ }^{,+, 1}$ David M. Antle, ${ }^{\S}$ Julie Côté, ${ }^{+, \S}$ Kathy Deroy, ${ }^{\|}$Nya Fraleigh, ${ }^{\mathrm{T}}$ \\ Karen Messing, ${ }^{\dagger, \ddagger}$ Lise Parent, ${ }^{\dagger, \#}$ Joey St-Pierre,, Cathy Vaillancourt, ${ }^{\dagger, \|}$ \\ and Donna Mergler ${ }^{\dagger,+}$ \\ *Medical Sciences Division, Northern Ontario School of Medicine, Sudbury, Ontario, Canada; \\ ${ }^{\dagger}$ Centre de Recherche Interdisciplinaire sur la Biologie, la Santé la Société, et l'Environnement \\ (CINBIOSE) and `Département des Sciences Biologiques, Université du Québec à Montréal, \\ Montréal, Québec, Canada; ${ }^{\S}$ Department of Kinesiology and Physical Education, McGill University, \\ Montréal, Québec, Canada; "Institut National de la Recherche Scientifique (INRS)-Institut Armand- \\ Frappier, Université du Québec, Laval, Québec, Canada; ${ }^{\mathbb{I}}$ Department of Biology, Laurentian \\ University, Sudbury, Ontario, Canada; and " Télé-université, Université du Québec, Montréal, \\ Québec, Canada
}

\begin{abstract}
In recent decades there has been an increasing recognition of the need to account for sex and gender in biology and medicine, in order to develop a more comprehensive understanding of biological phenomena and to address gaps in medical knowledge that have arisen due to a generally masculine bias in research. We have noted that as basic experimental biomedical researchers, we face unique challenges to the incorporation of sex and gender in our work, and that these have remained largely unarticulated, misunderstood, and unaddressed in the literature. Here, we describe some of the specific challenges to the incorporation of sex and gender considerations in research involving cell cultures and laboratory animals. In our view, the mainstreaming of sex and gender considerations in basic biomedical research depends on an approach that will allow scientists to address these issues in ways that do not undermine our ability to pursue our fundamental scientific interests. To that end, we suggest a number of strategies that allow basic experimental researchers to feasibly and meaningfully take sex and gender into account in their work. - Ritz, S.A., Antle, D. M., Côté, J., Deroy, K., Fraleigh, N., Messing, K., Parent, L., St-Pierre, J., Vaillancourt, C., Mergler, D. First steps for integrating sex and gender considerations into basic experimental biomedical research. FASEB J. 28, 4-13 (2014). www.fasebj.org
\end{abstract}

Key Words: transformed cell lines $\cdot$ primary cell cultures • animal models

Over THE PAST SEvERAL decades, it has become very clear that research conducted solely on male subjects cannot necessarily be extrapolated to females, and vice versa. Although most basic biomedical researchers are well aware of this, for the most part sex and gender $(\mathrm{s} / \mathrm{g})$ considerations

Abbreviations: s/g, sex and gender continue to be largely ignored unless the research concerns reproductive biology or sex-specific cancers, or where the primary purpose of the research is to discern whether there are sex differences for a given outcome. Even in these cases, however, the majority of the focus is on sex only, without consideration of gender. Outside of these areas, $\mathrm{s} / \mathrm{g}$ issues are not often addressed in basic experimental biomedical research $(1,2)$.

Although the terms "sex" and "gender" are often used interchangeably, in fact there is a useful conceptual distinction to be made between them: sex can be thought of as a biological attribute (such as those characteristics relating to genetics, physiology, anatomy, or reproduction) used to classify sexually reproducing animals (typically as males or females), while gender refers to the social processes that collectively influence the social roles, relationships, behaviors, power, or other traits that are culturally accorded to those classified as women/girls and men/boys (3-5). It should be noted that although we often talk about $\mathrm{s} / \mathrm{g}$ in terms of binaries, i.e., male/female, woman/man, boy/girl, and feminine/masculine, such dichotomies are artificial and fail to reflect the diversity of possible sexes and genders and the interactions between biological and social factors. A full discussion of these ideas is beyond the scope of this work, but for further reading, we recommend refs. $6-9$ as a starting point. Making a distinction between the concepts of sex and gender enables us to explicitly recognize that both biological and culturally specific social factors may have an important effect on biology and health, and that neither is necessarily fixed or determined (Fig. 1).

\footnotetext{
${ }^{1}$ Correspondence: Northern Ontario School of Medicine, East Campus-Laurentian University, 935 Ramsey Lake Rd., Sudbury, ON P3E 2C6, Canada. E-mail: sritz@nosm.ca doi: 10.1096/fj.13-233395
} 


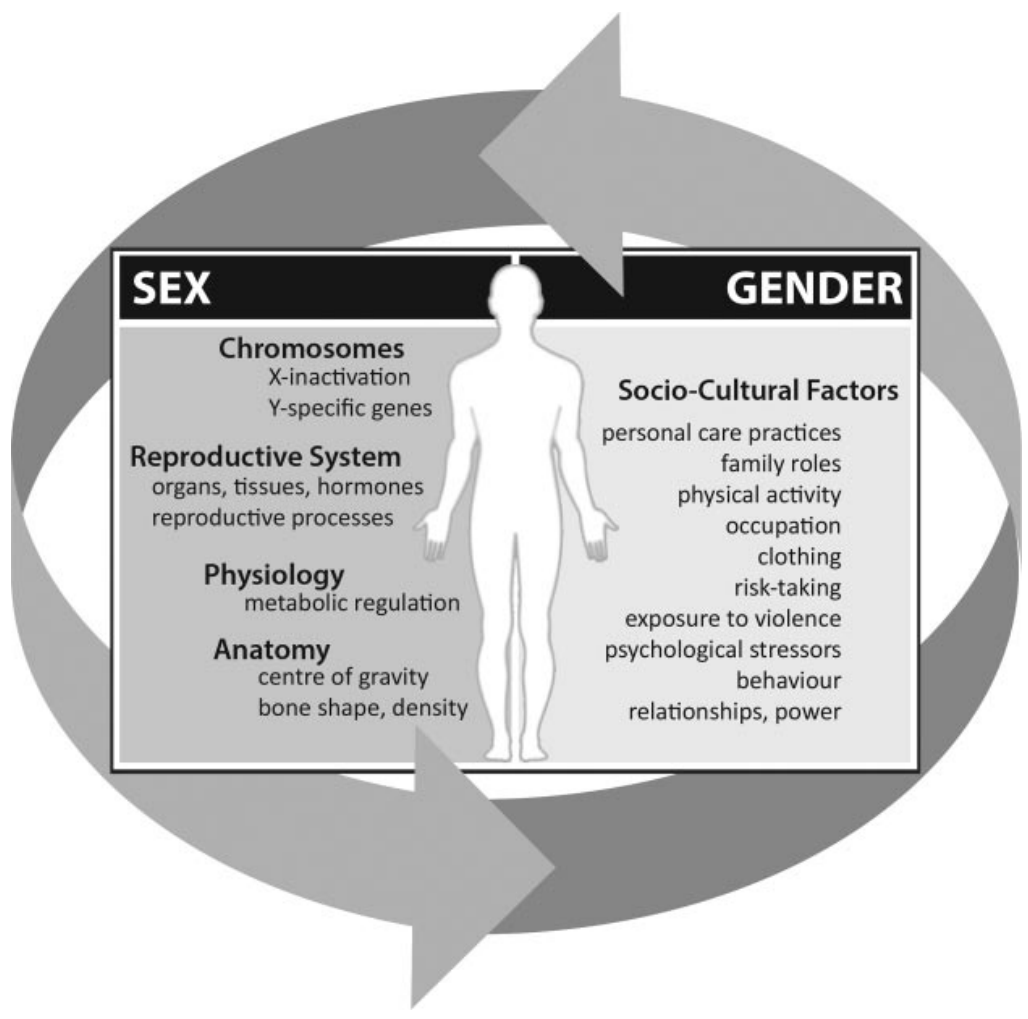

Figure 1. The distinction between sex and gender. Although often used interchangeably, sex and gender can usefully be distinguished from one another. Sex refers to those characteristics that are rooted in biological differences between male and female bodies, including chromosomal, genetic, anatomical, reproductive, and physiological traits. On the other hand, gender speaks to the social and cultural aspects of masculinity and femininity. Sex and gender are distinct concepts, but there is significant interaction between them, with aspects of biology influencing social dynamics and vice versa. Although sex and gender are often thought of as dichotomous variables that neatly divide people into males/men and females/women, in fact such classifications are not as clear and unambiguous as we tend to assume.

Of course there is no single recipe for the inclusion of $\mathrm{s} / \mathrm{g}$ considerations, and certainly the challenges in doing so differ between disciplines, methods, and paradigms: a researcher undertaking qualitative studies of men's experiences of prostate cancer will obviously use different means to consider $\mathrm{s} / \mathrm{g}$ issues than will a researcher trying to understand how $\mathrm{s} / \mathrm{g}$ might influence cell signaling pathways in vitro. Although there are many voices calling for researchers to attend to these kinds of questions, there are unique challenges to the incorporation of $\mathrm{s} / \mathrm{g}$ in laboratory-based experimental research, and a shortage of practical guidance about how to overcome these obstacles. Indeed, others have recognized that contemporary theorizing about $\mathrm{s} / \mathrm{g}$ in science is far more sophisticated than the tools we use to study it (1), and the basic sciences are lagging far behind the rest of the field in addressing $\mathrm{s} / \mathrm{g}$ issues.

In this paper, we will discuss some of the reasons why addressing $\mathrm{s} / \mathrm{g}$ issues in experimental biomedical science is not a straightforward proposition, and offer a number of suggestions for scientists who wish to begin to consider $\mathrm{s} / \mathrm{g}$ issues in their work. We have first-hand experience of these difficulties ourselves, and many of our suggestions are aimed at heightening awareness of $\mathrm{s} / \mathrm{g}$ considerations in laboratory research, rather than at the literal incorporation of $\mathrm{s} / \mathrm{g}$ into our experiments. For those scientists for whom sex or gender are already a major focus of the research, these recommendations may not be very helpful. However, for those among us who have not previously considered s/g in our work, it is our hope that these ideas will provide some starting points to help researchers gain a conceptual foothold in this important area. Our motivation is not political correctness, but rather to ensure that important phenomena are not overlooked that would be helpful in understanding biological mechanisms and processes, which may be undetected or misunderstood when $\mathrm{s} / \mathrm{g}$ is not addressed or when $\mathrm{s} / \mathrm{g}$ is addressed in overly simplistic terms that treat them as fixed binary traits.

\section{COMPLEXITIES OF $\mathrm{S} / \mathrm{G}$}

For the most part, basic scientists tend not to distinguish between the terms sex and gender, and use them interchangeably, despite the conceptual distinctions that can be made between them, as described above. The sex-gender distinction began to be articulated in the 1970s in an effort to challenge biological determinist views of the differences between men and women (5). Although use of the term gender in the scientific literature has increased dramatically over the past several decades $(10-12)$, it is usually used as though gender were "just a polite way to say sex" (1). Indeed, it is not hard to find instances in which rats, mice, guinea pigs, fish, plants, isolated cells, and body fluids, are described as having gender.

While it is relatively easy to make a distinction between the concepts of $\mathrm{s} / \mathrm{g}$, in practice we find that they are not as neatly separated as we might think. For example, Fausto-Sterling has pointed out that a trait like bone density is influenced both by sex-linked biological factors, such as sex hormones, and by gendered social dynamics; dress, occupation, and physical activity also play a role in determining bone density because they can create gendered variations in factors 
such as sun exposure and vitamin D synthesis, or weight-bearing activities (13). In addition, although we are quick to see the ways in which biological sex can shape the social practices of gender, it is also the case that social influences can affect biology; for example, social relationships can influence the function of the hypothalamic-pituitary-adrenal axis in response to stress (14), and engaging in nurturing behaviors can influence testosterone levels (15). Examples like these serve to remind us that observed $\mathrm{s} / \mathrm{g}$ differences are often not based purely in biology, but are the result of a complex interplay between the social and the biological. Indeed, when we attempt to unravel the interactions between biological factors and social experiences we often find "that it is impossible to separate out any one of these influences from another" (16). We frequently use the composite term "sex/gender" when discussing these issues because it reflects this complexity.

Despite the fact that it is often difficult to disentangle sex and gender, making a conceptual distinction between them is useful for basic scientists because it enables a more nuanced understanding of the ways that the biological and social effect on the phenomena of scientific interest to us (5). It also helps to reveal the ways in which our cultural beliefs about $\mathrm{s} / \mathrm{g}$ may influence our scientific theorizing. It is of crucial importance to recognize that attending to s/g considerations is not simply about seeking out differences between males/men and females/women, as all too often, any differences found between them are overinterpreted and strongly colored by our cultural biases about gender. In an illuminating example, Martin has described how gender stereotypes have affected the ways that scientists describe and understand reproductive biology, in some cases unconsciously interpreting the data so as to fit more easily with our cultural beliefs about gender roles (17). Similarly, Fine has comprehensively discussed the ways that cultural biases about men and women have infiltrated scientific theorizing about neurobiology (18). In another revealing case, associations between parity and incidence of melanoma in women were often attributed to pregnancy-related hormonal changes, until additional work demonstrated a similar relationship in men, suggesting that the relationship is more likely explained by the social conditions related to parity than to the biological effects of pregnancy $(5,19)$. With these examples in mind, it is clear that taking up $\mathrm{s} / \mathrm{g}$ in our research does not simply mean comparing females/women and males/men, but is a much larger enterprise; it's also about paying heed to the ways that $\mathrm{s} / \mathrm{g}$ may shape the way we think about our scientific questions.

\section{CHALLENGES TO ADDRESSING SEX IN EXPERIMENTAL LABORATORY RESEARCH}

There are a number of reasons why basic biomedical researchers tend not to address $\mathrm{s} / \mathrm{g}$ in their work. In some cases, $\mathrm{s} / \mathrm{g}$ issues are perceived as being irrelevant to the area of interest (though this is seldom actually the case). In others, although there may be awareness that $\mathrm{s} / \mathrm{g}$ considerations are relevant in some way, there is also a sense that examining $\mathrm{s} / \mathrm{g}$ in the experiments themselves is a distraction from the primary focus of the work. Even in research where the relevance of $\mathrm{s} / \mathrm{g}$ is clear, there are some practical barriers that may prevent us from incorporating it at the laboratory bench.

Setting gender aside for a moment, it is often suggested that scientists could address the question of sex simply by using both male and female cells or animals in our experiments. On the surface, this appears to be a simple and straightforward solution, but in practice such an approach often proves to be unfeasible or inadequate, and may even promote an overly simplistic and deterministic way of thinking about sex that overemphasizes and naturalizes difference.

\section{In vitro research}

For many of us working with human transformed cell lines, the advice to simply include male and female cells is not workable, since the process of establishing stable immortalized cell lines from human subjects inherently precludes the possibility that equivalent lines from male and female sources could ever be created. Although we may be able to create or obtain cell lines with similar characteristics from both male and female human subjects, such cell lines are likely to be significantly different from one another such that any differences observed between them could not be reliably ascribed to sex.

For example, the A549 cell line is commonly used as a model for airway epithelium in respiratory research. A549 cells are derived from the tumor of a male patient with lung carcinoma, and are known to be hypotriploid in their chromosome complement; in addition, although A549 cells were derived from a male patient, one or both Y chromosomes are absent in $40-50 \%$ of the cells (20). An airway epithelial cell line from a female patient, the HCC827 line, is also commercially available, but it is derived from a lung adenocarcinoma, and is known to have a mutation in the tyrosine kinase domain of the epidermal growth factor receptor (21). Attempts to compare these two cell lines as a way of addressing sex would obviously be misguided, as any differences observed could just as easily be related to idiosyncrasies introduced in the process of establishing the cell lines, to the mutations which led to tumor formation, or to the epigenetic effects of social factors such as socioeconomic status or ethnicity, rather than to the sex of the patients they were derived from. Moreover, we obviously should not expect that cells from a single male or female patient could ever appropriately represent all males or females. Thus, even where similar human male and female cell lines are available, this would be a highly dubious approach to addressing sex in these experimental contexts.

Given these limitations, some have suggested that such work with human cells might more appropriately 
be done in primary cultures, in which cells are isolated directly from body tissues and grown in vitro, so that cells could be obtained from a variety of male and female donors. Although this is theoretically possible, there are several problems that make this approach infeasible for many situations. For many cells of interest, the establishment of primary cultures is a very time-consuming and technically demanding procedure that does not always yield large numbers of cells. Moreover, because primary cells have a limited lifespan in vitro, and can only undergo a limited number of cell cycles before they stop dividing and become senescent, in order to do ongoing experiments with primary cultures one has to freshly re-isolate primary cells from tissue on an ongoing basis. This may be relatively simple when the cells of interest are easily obtained in large numbers, but many cells of interest to researchers are significantly more difficult to obtain, especially if they represent specific rare cell populations. The human and financial resources required to replace transformed cell lines with primary cultures are wholly beyond the means of the vast majority of researchers.

However, even if resources were not a limiting factor for using primary cell cultures (or if we were able to shift our experimental models to use cells from inbred animals where it may be possible to create equivalent male and female cell lines), we argue that any straight-up comparison of cultured male and female cells oversimplifies the question of sex because of the limitations of the in vitro environment itself. Discussions about incorporating sex into such experiments has tended to focus on the source of the materials (that is, whether the cells are from male or female donors). But sex is not a fixed property of cells themselves - it is also a dynamic and changeable property of the body (12). When cells are removed from the body and placed into culture, they do not necessarily take everything of what it is to be male or female with them. In the body, cells are subject to myriad complex interactions between different cell types, sex hormones, stress hormones, neurotransmitters, nutrients, cytokines, coenzymes, neural input, and environmental exposures, each of which will vary according to the sex of that body and its gendered social experiences (which will also interact with many other kinds of social influences, such as socioeconomic status or experiences of stigma and discrimination); when placed into culture, the complex input of the sexed and gendered body largely disappears (Fig. 2).

In vitro, the only sex differences these isolated cells can be expected to manifest are those that are generated at the level of the individual cell (e.g., arising directly and solely from their chromosomal complement), or that have conditioned the cell in some permanent or persistent way (e.g., through DNA methylation). It is important to recognize this limitation of the in vitro environment in interpreting any findings where cultures of male and female cells are involved. It is possible or even likely that conditions present in male or female bodies (such as the differential presence of sex hormones) would in fact cause cells to respond differently to the same stimulus in vivo; however, when the cells exist in identical and static culture conditions, such differences may not be apparent. Thus, if no differences are observed between the responses of male and female cells in vitro, one must resist the temptation to conclude that sex does not affect the phenomenon of interest. Conversely, it is also tempting to surmise that any differences observed in vitro must be due to biologically based differences between males and females, but we must be cautious about rushing to this

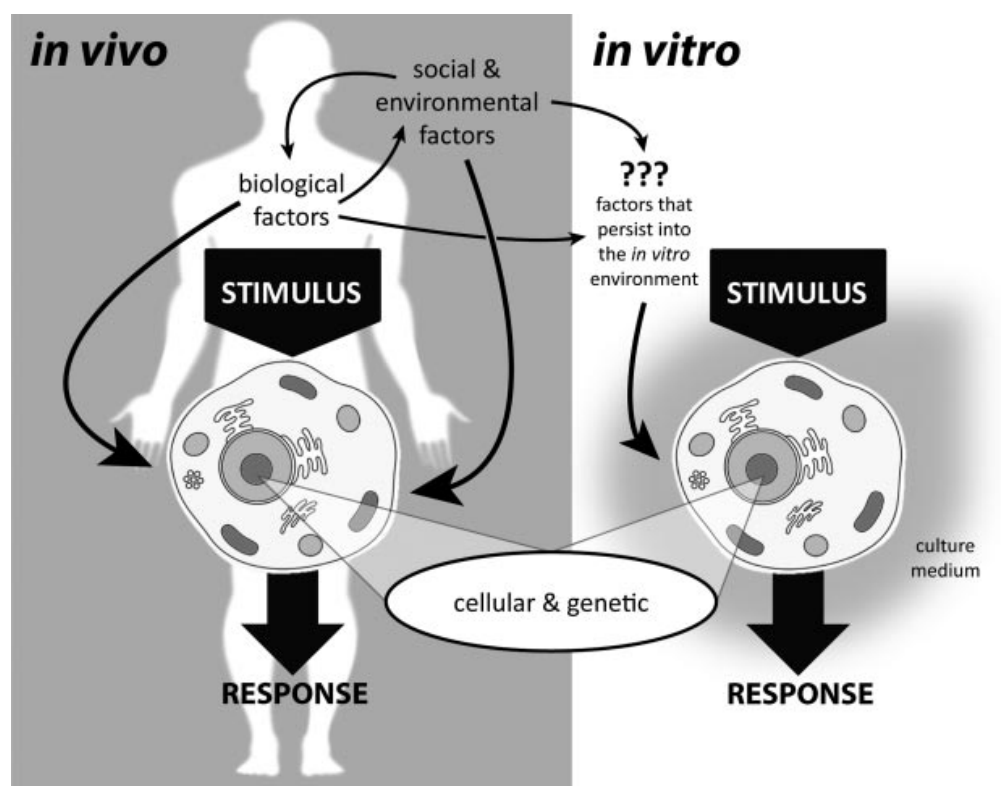

Figure 2. Limitations of in vitro experimentation for examining the influence of sex on biological phenomena. In the body, a cell's response to a given stimulus will be modulated by the interplay of a variety of factors. Biological factors may influence the cell's behavior, both due to properties intrinsic to the individual cell itself (such as its chromosomal complement, or cellular mechanisms related to $\mathrm{X}$ chromosome inactivation), and sex-related properties of the body (such as hormonal influences or metabolic status). At the same time, environmental and social experiences can also influence the cell's activities by moderating the production of neurotransmitters and hormones, or through exposure to exogenous agents, for example. In contrast, when a cell is isolated from its bodily context and placed into culture, this network of $\mathrm{s} / \mathrm{g}$ influences is disrupted. Those differences that arise directly from the chromosomal complement of the cells will transfer into the in vitro environment, and cells may have become conditioned by their sexed/ gendered experiences in ways that may or may not persist in vitro (through mechanisms such as DNA methylation, for example). Thus, even if male and female cells are used, we cannot assume that the cell's response in culture reflects the true effect of $\mathrm{s} / \mathrm{g}$ influences. 
conclusion as well. It is conceivable that cells could behave differently in vitro as a result of socially produced differences in exposure that persistently conditioned the cells (as a result of DNA methylation or other mechanisms that could modify gene expression). Thus, we cannot assume that the mere inclusion of male and female materials in in vitro research will allow us to develop an adequate understanding of $\mathrm{s} / \mathrm{g}$ issues.

\section{Animal research}

Unlike cell work, where it may not be possible to get male and female materials, obtaining equivalent male and female research animals is often as simple as ordering them from the supplier, especially for commonly used species such as mice and rats. However, this is not universally the case; for some transgenic animals or disease models in animals, male or female animals may not be viable, or the disease model may not manifest in one of the sexes. In addition, in studies of immature animals or species without obvious signifiers of sex, it can be complicated to determine the sex of individual animals without undertaking genotyping.

In most fields of study, male animals are used more often than females (with some notable exceptions), and few studies incorporate both males and females (2, 22). Cost is probably part of the reason for this. Including male and female animals in an experiment would at least double the human and financial resources required; some recommendations go even further, suggesting that in mouse studies 4 different groups of female mice should be used to account for the stages of the murine estrus cycle as well (23). Although some may argue that the investment is justified to address the knowledge gap, given the relative scarcity of research funding it is not surprising that most biomedical scientists (and peer reviewers, for that matter) find it hard to justify this approach on a broad basis without a high likelihood of benefit.

Even where it is feasible to use both male and female animals experimentally, the living conditions of the animals may create some confounding effects. With laboratory rodents, male and female animals are usually segregated unless they are being bred. Due to the propensity of male rodents to fight with one another, they are typically housed at a lower density than females, which could have significant consequences for the biological phenomena under study. For example, male mice housed alone may have to expend more energy maintaining body temperature than females housed in groups (who often sleep clustered together), which could cause differences in parameters such as caloric intake, muscle activity, metabolic rate, fat distribution, or body size, with a plethora of potential downstream effects on all bodily and cellular activities; this could create a situation in which sex differences were found as a result of artifacts produced by the experimental housing conditions. Other social phenomena with biological effects have been documented as well, such as the "barbering" that occurs in many strains of laboratory mice (24), or estrus suppression in female mice housed together $(25,26)$. Housing male and female animals under identical conditions would be ideal, but because the social stressors for males housed together would likely be different than for females (again creating the possibility of artifacts), this would essentially require that all animals be caged individually (which itself could be a stressor for animals who normally live in social groups). Although simple to implement in theory, in practice this would have significant financial implications and require major infrastructural adaptations in most animal research facilities in order to accommodate such a change.

It should be evident by now that the incorporation of sex considerations into laboratory-based cell and animal research is not necessarily as simple and straightforward as it might at first seem, and may have significant practical and financial implications. In addition, an approach to sex that frames it as a simple dichotomy between male and female probably tends to overemphasize differences between them, and takes for granted that sex is a binary and fixed biological trait (a view that has been challenged by many scholars) (7, 9, 27). Although many biomedical researchers acknowledge the limitations of research that does not account for sex, faced with these kinds of challenges, it is not surprising that most of us opt not to go down that path experimentally.

\section{CAN GENDER BE ADDRESSED AT ALL IN LABORATORY RESEARCH?}

Given that addressing sex in laboratory-based research is more challenging than it may first appear, incorporating gender considerations is likely to be even more complex. For starters, gender is an inherently cultural construct, and gender practices in human societies probably cannot be simplistically equated to social dynamics among nonhuman subjects. Behaviors such as nurturing and aggression should not be assumed to be equivalent between experimental animals and in humans, as the causes and results of such behaviors may be very different, not to mention the fact that our own biases may lead us to interpret animal behavior in anthropocentric ways (28).

But there are additional reasons why gender is difficult to address that are not about bias or feasibility. Probably the most fundamental difficulty in incorporating gender into basic experimental research goes right to the heart of the philosophical basis of knowledge building that underpins experimental laboratory research. The classic framework under which most experimental studies are conducted is based on empirical and systematic observation and the attempt to control all relevant variables save for the outcome of interest, with a view to minimizing contextual biases. Thus, the incorporation of an inherently complex construct like gender, which is fundamentally about social context, may be difficult to reconcile with a positivistic scientific 
framework that aspires to reduce complexity and control as many variables as possible.

Having contemplated this difficulty at length, we are skeptical that gender itself can be directly addressed in most experimental laboratory research. Although it is conceivable that we might be able to model certain aspects of human gender in experimental settings, it is hard to imagine doing so in authentic and meaningful ways that do not trivialize human gender practices. Certainly, we would need to exercise a strong vigilance about how our own stereotypes about sex and gender might influence such models and the interpretations of data emerging from them $(17,28)$.

\section{FIRST STEPS: A BASIC TOOLBOX FOR INTEGRATING S/G IN EXPERIMENTAL RESEARCH}

Ultimately, experimental scientists are unlikely to embrace the incorporation of $\mathrm{s} / \mathrm{g}$ issues into our work if we perceive it as undermining the central focus of our research, nor would it would be desirable to have us all comparing males and females in search of differences. We agree with Sharman and Johnson, that the goal is "to create a framework for encouraging [biomedical] researchers to engage in a considered, theoretically sound exploration of difference as it may or may not pertain to their research designs" (1).

In our work in the laboratory, we have struggled to find ways to address $\mathrm{s} / \mathrm{g}$ in our research, and we appreciate that exhortations to include $\mathrm{s} / \mathrm{g}$ considerations are not terribly useful in the absence of feasible approaches that are compatible with the realities of laboratory-based experimental research. We know that advice to "just include male and female cells or animals" is simply not adequate.

Some granting agencies and journals are now requiring applicants and authors to answer questions about $\mathrm{s} / \mathrm{g}$ and conform to requirements to include s/g considerations in their work, which can be challenging for many basic scientists. We have identified a toolbox of actions that laboratory researchers can take to begin the process of addressing s/g issues (Fig. 3). These baby steps are small, reasonable actions that can be undertaken without imposing unreasonable resource burdens or diverting the focus of the research. Nearly any researcher, regardless of the experimental system, disease model, or discipline, can incorporate one or more of these approaches. Most of these suggestions do not require us to literally incorporate $\mathrm{s} / \mathrm{g}$ into the experiments, but can still serve to raise the profile of $\mathrm{s} / \mathrm{g}$ issues in biomedical research broadly, and foster a richer scientific dialogue that more readily recognizes the relevance of $\mathrm{s} / \mathrm{g}$ for many fields of study.

\section{Step 1: Develop an active knowledge of $\mathrm{s} / \mathrm{g}$ issues}

Obviously, we will be unable to address $\mathrm{s} / \mathrm{g}$ issues in our research if we do not have a solid handle on the
Sex/Gender Toolbox for Experimental Scientists

Develop your knowledge of S/G issues

- Do a ca reful literature review. Are there known sexdifferences or gender disparities for the phenonmenon of interest?

- Avoid using the terms 'sex' and 'gender' interchangeably in you r writing.

\section{Discuss S/G where appropriate}

- Always report the sex of the cells, tissues, a nimals, or subjects you are using.

- If using one sexonly, justify why, and note the limitations in your discussion.

- Al ways discuss the possible s/g implications of your findings.

\section{Introduce a small intervention}

- Do a small pilot experiment to examine the influence of some element ofs/g in your model system:

- Add a hormone to one of your cultures.

- Include male and female a nimalsin the key experimental groups.

- Report what you find, whether sex differences a re observed or not.

\section{Raise the profile of $S / G$ issues}

- As a reviewer, ensure that applicants/authors identify and justify the sex of the materials used, and make sure that the terms 'sex' and 'gender' are used a ppropriately.

- Ask questions of colleagues and trainees: have they considered whether s/g issues might be relevant to theirwork?

Figure 3. First steps for integrating $\mathrm{s} / \mathrm{g}$ in experimental biomedical research. Although it may not be feasible to literally incorporate $\mathrm{s} / \mathrm{g}$ considerations into our experimental work, there are a number of ways to address $\mathrm{s} / \mathrm{g}$ issues in our work that do not require us to fundamentally alter our research focus.

concepts of $\mathrm{s} / \mathrm{g}$ themselves. As discussed earlier, the biomedical sciences literature is rife with instances where gender is used as a synonym for sex. This tendency is problematic because it serves to reinforce the perception that differences between men and women are inevitable and based in hard-wired biological differences, thereby obscuring the powerful influence of social factors on health. Thus the first step in enhancing the way we address $\mathrm{s} / \mathrm{g}$ issues in biomedical research is simply to ensure that we understand the terms and are using them appropriately. As previously 
discussed in greater detail, sex refers to traits thought to be primarily based in biological characteristics that are key features of making distinctions between sexually reproducing animals (typically as male or female), whereas gender should be used when discussing characteristics that are primarily related to sociocultural norms. As such, we would suggest that the use of the term gender should be reserved almost exclusively to refer to human beings (12), and that it is not generally sensible to talk about the gender of a mouse or a cell (even if that cell came from a human being), with rare exceptions. In cases where the biological and social contributions are hard to discern, it may be useful to simply use "sex/gender."

In addition, it is impossible to address the $\mathrm{s} / \mathrm{g}$ issues in our research if we are not familiar with what is already known on the subject within our own disciplines. Often, basic scientists claim that $\mathrm{s} / \mathrm{g}$ is not pertinent to their work (1), but the literature shows that it is rare that $\mathrm{s} / \mathrm{g}$ issues are truly irrelevant for most biomedical research. The field of immunology serves as a stark example of this. Although it has been very well documented that sex has a profound influence on immunological function (29-34), Beery and Zucker (2) found that less than $10 \%$ of immunology articles reported using animals from both sexes, and more than $60 \%$ of articles failed to specify the sex of the animals that were used. A disparity of this magnitude suggests a major blind spot on the part of immunologists with respect to the importance of $\mathrm{s} / \mathrm{g}$ issues in their field (35). The disjuncture was less striking in other fields, but there were marked imbalances in the use and reporting of data with respect to sex in most other disciplines analyzed (2).

A vast number of cellular, physiological, and behavioral processes are known to be influenced by factors that vary with $\mathrm{s} / \mathrm{g}$. A quick search of your research area in your favorite database using keywords such as "sex," "gender," or "sex differences," or perhaps other keywords relating to specific aspects of s/g, such as "estrogen," "Y chromosome," or "occupation," will often dispel the perception that $\mathrm{s} / \mathrm{g}$ issues are irrelevant. At most academic institutions, librarians will be very happy to assist in developing a search strategy to identify the literature of interest. Excellent review articles are available as succinct entry points to explore these issues for many scientific disciplines (see, for example, refs. 3650). Regardless of whether we act on this knowledge experimentally, making ourselves aware of these pockets of literature related to our disciplines will enable an informed evaluation of the relevance of $\mathrm{s} / \mathrm{g}$ for our work, and will deepen our understanding of our research areas more generally.

\section{Step 2: Address $\mathbf{s} / \mathrm{g}$ issues when reporting and interpreting findings}

When working with biological materials, we should always report the sex of the cells, tissues, or animals we are working with (12); this may seem obvious, but Beery and Zucker's analysis of the literature from 2009 showed that in some fields (particularly general biology, immunology, neuroscience, and physiology), significant proportions of nonhuman animal studies failed to report the sex of the animals used (2), and work in transformed cell lines rarely makes note of this. For transformed cell lines or primary cell lines ordered from commercial suppliers, it is frequently the case that information about the sex of the donor is not available (12); in these instances, it is still worthwhile to note in the methods section that the sex of the cells was not known.

We could consider also including a rationale for the sex of the cells or animals that were used. Such justifications do not need to be profound, but would serve to increase the transparency of our decision-making around these issues. In many cases, it may be that most of the previous research in a given field has used one sex, and so we have used the same sex in order to allow comparisons to previous work. In other cases, perhaps the primary concern is to avoid the effects of hormonal fluctuations associated with estrus cycling. The simple act of acknowledging these realities is a worthwhile step forward in our discourse on $\mathrm{s} / \mathrm{g}$.

The natural extension of reporting and justifying the sex of the cells or animals used in one's investigations is to introduce some discussion of the implications of doing so, just as we acknowledge the limitations of our experimental models in other ways. In animal work, where only one sex was used, consider including a short paragraph in the discussion that acknowledges this limitation of the work, referencing the literature with respect to existing evidence relating to sex effects on the phenomenon studied and how this may color the interpretation of your findings.

Even though it may be difficult or even impossible to address gender directly in our experiments, there may well be gender issues of relevance for the way we contextualize our work. For example, in environmental health it is often the case that the prevalence of exposure to chemicals varies with gender (such as those associated with particular occupations or household activities); a researcher conducting experimental work related to the subcellular pathways activated by such a chemical probably cannot address gender in the experiment itself, but gendered exposure patterns would certainly be relevant to bring up in their discussion of the data. In fact, this kind of discussion may be the only reasonable way to address gender for most of us doing experimental laboratory research.

\section{Step 3: Introduce a small intervention and report what you find}

Although not every experiment needs to incorporate sex, it is often informative to conduct some small pilot experiments to directly examine whether sex might influence the experimental systems we use.

Noting the substantial limitations to addressing sex in cell culture systems discussed previously, it is not at 
all simple to capture the totality of what we mean by sex in vitro, but we can think about ways to incorporate aspects of sex into these systems. For example, one might add a single gonadal hormone, such as estrogen or testosterone, to the cultures to examine its effect on the outcome of interest. Where your expansion or isolation procedures leave you with an excess number of cells beyond what is strictly required for the experiment, rather than dispose of these extra cells, consider plating an additional few wells of your key control and experimental conditions and add the hormone to these wells. Among ourselves, N.F. and S.A.R. have used this approach in primary cultures of human T-helper cells exposed to nickel, and found that the presence of estrogen indeed affected the profile of cytokines secreted by activated cells.

Similarly, in animal studies, though it is unlikely that we will be able to use male and female animals in every experiment, including both sexes even one time can be revealing. Since the purpose here is not to generate definitive and comprehensive data, not all time points or experimental groups need to be covered, and the number of animals need not be as large as if you were trying to power the experiment sufficiently for statistical analysis. If you usually use males, adding a small group of females for the main control condition and the main experimental condition may be adequate to give you a sense of whether or not there are notable differences between males and females for any of the major outcomes.

In either case, this need not be a long-term commitment to continue experimentation in this vein, but even a cursory examination of whether your outcome of interest is likely to be influenced by sex or sex-related factors will often be enlightening. Even if you choose not to pursue the findings any further, it is extremely valuable to report what you find in any related publications, even if the data is not statistically significant or provided in a figure. A succinct statement in the results or discussion section describing the outcome, with "data not shown," can serve to inform others of the potential presence or absence of a sex-related influence. In fact, reporting negative data is especially valuable in this regard; since positive data are more likely to be published than negative findings (the so-called file-drawer problem; ref. 51), documenting the absence of a sex difference helps to ensure that difference is not artificially overemphasized in the literature.

At the same time, it is extremely important not to bring an overly simplistic framing of $\mathrm{s} / \mathrm{g}$ to the interpretation of such experiments. For example, we must be cognizant that hormone levels vary widely across the life span, and specific hormones are not exclusively present in one sex; that male and female mice are not human men and women; that within the categories male and female, there is enormous variation for most measures; and that not all individuals fit neatly into one of these categories. Our cultural stereotypes make it all too easy to fall into the trap of making "men are from
Mars and women are from Venus" sorts of generalizations, or mistaking correlation for causation; attending to $\mathrm{s} / \mathrm{g}$ issues appropriately means that we must make conscious efforts to resist these tendencies.

\section{Step 4: Use your influence to raise the profile of $\mathrm{s} / \mathrm{g}$ issues}

Perhaps the simplest thing that can be done is to create a more prominent discourse around $\mathrm{s} / \mathrm{g}$ issues in the basic sciences by raising the issue more frequently. At present, most of the discussions are limited to pockets of research directly focused on s/g issues, and rarely get voiced outside these domains. Our varied roles as scientists give us many opportunities to change this.

The process of peer review provides an ideal forum to raise awareness about $\mathrm{s} / \mathrm{g}$ and prompt our colleagues to think about these matters. When reviewing papers or grant proposals, it is fair to request that authors or applicants identify the sex of the cells or animals used, seek a rationale for the use of one sex only, or encourage the authors to include a brief discussion of the limitations introduced by doing so. We can flag instances where the terms sex or gender are used improperly, explaining which term is appropriate and why. Of course such queries should always be constructive and posed in the spirit of widening the discussion of $\mathrm{s} / \mathrm{g}$ issues in biomedical sciences, as the whole point is to engage our colleagues, not to put them on the defensive.

Another simple intervention is to make a point of asking questions about $\mathrm{s} / \mathrm{g}$ at conferences, seminars, rounds, or other venues where scientists give talks, and asking the speakers whether they have considered s/g issues in their research. Several of us have started making a habit of this, and it often generates quite vibrant and constructive discussion about gaps in the field.

Many of us are also teachers and mentors, and these roles offer us additional opportunities to raise the profile of $\mathrm{s} / \mathrm{g}$ at a number of points. In formal classroom settings, one might consider including a lecture or session, incorporating an assignment, or making ongoing references to $\mathrm{s} / \mathrm{g}$ issues as appropriate; this might be an ideal forum in which to introduce students to the distinction between gender and sex. An excellent assignment for a graduate course or topic for a comprehensive examination would be to ask students to review the literature on $\mathrm{s} / \mathrm{g}$ in their field. Where we are supervising trainees in the lab, we can encourage a consideration of $\mathrm{s} / \mathrm{g}$ issues by prompting students to include a review of $\mathrm{s} / \mathrm{g}$ issues in their writing, or asking relevant questions during lab meetings. If you are looking for a project for a summer student or undergraduate thesis student, it might be the ideal opportunity to conduct the kinds of pilot experiments described above, and having that student undertake the accompanying literature review on $\mathrm{s} / \mathrm{g}$ issues as well. Undoubtedly our own appreciation of $\mathrm{s} / \mathrm{g}$ issues will be enhanced at the same time. 
These are simple interventions, but if taken up broadly, the effect could be quite profound. By making use of these opportunities to raise $\mathrm{s} / \mathrm{g}$ issues in the context of our day-to-day work, we can begin to build a larger conversation throughout the biomedical sciences about the relevance of $\mathrm{s} / \mathrm{g}$ for our research.

\section{FINAL THOUGHTS}

Compared to other fields, the experimental biomedical sciences have been slow to take up s/g considerations. Our objective in this article has been to articulate the challenges of addressing $\mathrm{s} / \mathrm{g}$ issues in laboratory-based research, and to identify ways of acknowledging their relevance in the face of the realities of experimental science, which create real limitations on what we are able to do, both practically and conceptually. We believe the basic toolbox we have offered demonstrates that there are ways to meaningfully take $\mathrm{s} / \mathrm{g}$ into account without diverting focus from our primary scientific interests, and without slipping into the traps of simplistic determinism or overemphasizing difference. We have deliberately taken this first-steps approach in order to be useful to a broad swath of researchers who otherwise might not consider $\mathrm{s} / \mathrm{g}$ at all. For those who want to integrate $\mathrm{s} / \mathrm{g}$ considerations more intensively in their work, there are a number of excellent resources available (for example, refs. 3, 4, 52).

Experimental biomedical research makes profoundly valuable contributions to the enterprise of building knowledge about the functioning of biological systems, and one of its greatest strengths is the ability to systematically test hypotheses through carefully controlled experiments. Therefore we need to judiciously consider the delicate balance between what might be gained or lost by introducing additional complexity into our experiments. Addressing $\mathrm{s} / \mathrm{g}$ issues in this context is anything but simple, although the challenges in doing so are not always apparent to those without first-hand knowledge of the realities of the laboratory. This complexity is not an excuse for ignoring $\mathrm{s} / \mathrm{g}$ for our research. Rather, this is a call to recognize that addressing s/g considerations in experimental biomedical research need not distract from, but can actually serve to enrich, our primary scientific interests.

The authors thank Marie-Eve Rioux-Pelletier, coordinator of the Canadian Institute of Health Research (CIHR) Team in Gender, Environment, and Health, for invaluable administrative support. The authors also thank all members and associates of the CIHR Team in Gender, Environment, and Health for conversations that have helped them to elucidate these ideas, and to Dr. Deboleena Roy, who offered intellectually generous and constructive critiques on the original version of this manuscript. Financial support for the CIHR Team in Gender, Environment, and Health was provided by an Emerging Team grant from the CIHR Institute of Gender and Health (GTA92108).

\section{REFERENCES}

1. Sharman, Z., and Johnson, J. (2012) Towards the inclusion of gender and sex in health research and funding: an institutional perspective. Soc. Sci. Med. 74, 1812-1816

2. Beery, A. K., and Zucker, I. (2011) Sex bias in neuroscience and biomedical research. Neurosci. Biobehav. Rev. 35, 565-572

3. Gendered Innovations Project (2011) Gendered Innovations in Science, Health E Medicine, Engineering, and Environment. Accessed June 6, 2013 at http://genderedinnovations.stanford.edu

4. Canadian Institutes of Health Research Institute of Gender and Health (2012) What a Difference Sex and Gender Make: a Gender, Sex and Health Research Casebook, Canadian Institutes of Health Research, Ottawa, ON, Canada

5. Krieger, N. (2003) Genders, sexes, and health: what are the connections-and why does it matter? Int. J. Epidemiol. 32, $652-657$

6. Connell, R. (2009) Gender: in World Perspective, Polity, Cambridge, UK

7. Delphy, C. (1993) Rethinking sex and gender. Women's Studies Int. Forum 16, 1-9

8. Fausto-Sterling, A. (2012) Sex/Gender: Biology in a Social World, Routledge, New York

9. Epstein, S. (2004) Bodily differences and collective identities: the politics of gender and race in biomedical research in the United States. Body Society 10, 183-203

10. Haig, D. (2004) The inexorable rise of gender and the decline of sex: social change in academic titles, 1945-2001. Arch. Sex. Behav. 33, 87-96

11. Walker, P. L., and Cook, D. C. (1998) Brief communication: gender and sex: vive la difference. Am. J. Phys. Anthropol. 106, 255-259

12. Wizeman T. M., and Pardue, M.-L. (eds) (2001) Exploring the Biological Contributions to Human Health: Does Sex Matter? National Academy Press, Washington, DC

13. Fausto-Sterling, A. (2005) The bare bones of sex: part 1-sex and gender. Signs J. Women Cult. Soc. 30, 1491-1527

14. Hostinar, C. E., Sullivan, R. M., and Gunnar, M. R. (2013) Psychobiological mechanisms underlying the social buffering of the hypothalamic-pituitary-adrenocortical axis: a review of animal models and human studies across development. [E-pub ahead of print] Psychol. Bull. PMID: 23607429

15. Van Anders, S. M., Tolman, R. M., and Volling, B. L. (2012) Baby cries and nurturance affect testosterone in men. Horm. Behav. 61, 31-36

16. Rogers, L. J. (2011) Sex differences are not hardwired. In Gender and the Science of Difference: Cultural Politics of Contemporary Science and Medicine (Fisher, J. A., ed) pp. 27-42, Rutgers University Press, New Brunswick, NJ, USA

17. Martin, E. (1991) The egg and the sperm: how science has constructed a romance based on stereotypical male-female roles. Signs 16, 485-501

18. Fine, C. (2010) Delusions of Gender: How Our Minds, Society, and Neurosexism Create Difference, W. W. Norton, New York

19. Kravdal, O. (1995) Is the relationship between childbearing and cancer incidence due to biology or lifestyle? Examples of the importance of using data on men. Int. J. Epidemiol. 24, 477-484

20. American Type Culture Collection (2013) Product Sheet: A549 (ATCC CCL-185), American Type Culture Collection, Manassas VA, USA

21. American Type Culture Collection (2013) Product Sheet: HCC827 (ATCC CRL-2868), American Type Culture Collection, Manassas VA, USA

22. Zucker, I., and Beery, A. K. (2010) Males still dominate animal studies. Nature 465, 690

23. Gendered Innovations in Science, Health \& Medicine, Engineering, and Environment (2011) Animal Research: Designing Health $\mathcal{E}$ Biomedical Research. Accessed June 6, 2013 at http:// genderedinnovations.stanford.edu/case-studies/animals.html

24. Kalueff, A. V., Minasyan, A., Keisala, T., Shah, Z. H. and Tuohimaa, P. (2006) Hair barbering in mice: implications for neurobehavioural research. Behav. Processes 71, 8-15

25. Champlin, A. K. (1971) Suppression of oestrus in grouped mice: the effects of various densities and the possible nature of the stimulus. J. Reprod. Fertil. 27, 233-241 
26. Ma, W., Miao, Z., and Novotny, M. V. (1998) Role of the adrenal gland and adrenal-mediated chemosignals in suppression of estrus in the house mouse: the lee-boot effect revisited. Biol. Reprod. 59, 1317-1320

27. Petersen, A. (1998) Unmasking the Masculine: "Men" and "Identity" in a Sceptical Age, Sage, Thousand Oaks, CA, USA.

28. Karlsson Green, K., and Madjidian, J. A. (2011) Active males, reactive females: stereotypic sex roles in sexual conflict research? Anim. Behav. 81, 901-907

29. Libert, C., Dejager, L., and Pinheiro, I. (2010) The X chromosome in immune functions: when a chromosome makes the difference. Nat. Rev. Immunol. 10, 594-604

30. Fish, E. N. (2008) The X-files in immunity: sex-based differences predispose immune responses. Nat. Rev. Immunol. 8, 737-744

31. Martocchia, A., Stefanelli, M., Cola, S., and Falaschi, P. (2011) Sex steroids in autoimmune diseases. Curr. Top. Med. Chem. 11, 1668-1683

32. Wilder, R. L. (2002) Neuroimmunoendocrinology of the rheumatic diseases: past, present, and future. Ann. N. Y. Acad. Sci. 966, 13-19

33. Verthelyi, D. (2001) Sex hormones as immunomodulators in health and disease. Int. Immunopharmacol. 1, 983-993

34. McCombe, P. A., Greer, J. M., and Mackay, I. R. (2009) Sexual dimorphism in autoimmune disease. Curr. Mol. Med. 9, 10581079

35. Klein, S. L. (2012) Immune cells have sex and so should journal articles. Endocrinology 153, 2544-2550

36. Pennell, L. M., Galligan, C. L., and Fish, E. N. (2012) Sex affects immunity. J. Autoimmun. 38, J282-J291

37. Fairweather, D., Cooper, L. T., Jr., and Blauwet, L. A. (2013) Sex and gender differences in myocarditis and dilated cardiomyopathy. Curr. Probl. Cardiol. 38, 7-46

38. Klein, S. L. (2012) Sex differences in prophylaxis and therapeutic treatments for viral diseases. Handb. Exp. Pharmacol. 499-522

39. Krause, D. N., Duckles, S. P., and Pelligrino, D. A. (2006) Influence of sex steroid hormones on cerebrovascular function. J. Appl. Physiol. 101, 1252-1261
40. Regitz-Zagrosek, V., Dworatzek, E., Kintscher, U., and Dragun, D. (2013) Sex and sex hormone-dependent cardiovascular stress responses. Hypertension 61, 270-277

41. Magkos, F., Wang, X., and Mittendorfer, B. (2010) Metabolic actions of insulin in men and women. Nutrition 26, 686-693

42. Waxman, D. J., and O'Connor, C. (2006) Growth hormone regulation of sex-dependent liver gene expression. Mol. Endocrinol. 20, 2613-2629

43. Demyanets, S., and Wojta, J. (2012) Sex differences in effects and use of anti-inflammatory drugs. Handb. Exp. Pharmacol. 443-472

44. Campesi I, Fois, M., and Franconi, F. (2012) Sex and gender aspects in anesthetics and pain medication. Handb. Exp. Pharmacol. 265-278

45. Spoletini I, Vitale, C., Malorni, W., and Rosano, G. M. C. (2012) Sex differences in drug effects: interaction with sex hormones in adult life. Handb. Exp. Pharmacol. 91-105

46. Durdiakova, J., Ostatnikova, D., and Celec, P. (2011) Testosterone and its metabolites-modulators of brain functions. Acta Neurobiol. Exp. (Wars.) 71, 434-454

47. Baylis, C. (2012) Sexual dimorphism: the aging kidney, involvement of nitric oxide deficiency, and angiotensin II overactivity. J. Gerontol. A Biol. Sci. Med. Sci. 67, 1365-1372

48. Vina, J., Gambini, J., Lopez-Grueso, R., Abdelaziz, K. M., Jove, M., and Borras, C. (2011) Females live longer than males: role of oxidative stress. Curr. Pharm. Des. 17, 3959-3965

49. Manole, M. D., Tehranian-DePasquale, R., Du, L., Bayir, H., Kochanek, P. M. and Clark, R. S. B. (2011) Unmasking sexbased disparity in neuronal metabolism. Curr. Pharm. Des. 17, 3854-3860

50. Barrett, E. L. B., and Richardson, D. S. (2011) Sex differences in telomeres and lifespan. Aging Cell 10, 913-921

51. Rosenthal, R. (1979) The file drawer problem and tolerance for null results. Psychol. Bull. 86, 638-641

52. Oliffe, J. L., and Greaves, L. (2012) Designing and Conducting Gender, Sex, and Health Research, Sage, Thousand Oaks, CA, USA

Received for publication June 17, 2013. Accepted for publication September 12, 2013. 